\title{
Production of charmed mesons and charmonium states in $B$ decays at Belle
}

\section{Olga Grzymkowska*}

The H. Niewodniczanski Institute of Nuclear Physics Polish Academy of Sciences

E-mail: olga.grzymkowska@ifj.edu.pl

Belle recently performed extensive studies of charmed and charmonium states from $B$ decays utilizing its $711 \mathrm{fb}^{-1}$ data set collected at $\Upsilon(4 S)$ resonance. We present here the results of three recent publications: arXiv:1504.02637, arXiv:1509.03363, arXiv:1505.03362. In the first publication improved measurements were reported of the product branching fractions $\mathscr{B}\left(B^{+} \rightarrow \overline{D^{0}} D_{s 0}^{*+}(2317)\right) \times \mathscr{B}\left(D_{s 0}^{*+}(2317) \rightarrow D_{s}^{+} \pi^{0}\right)=\left(7.8_{-1.2}^{+1.3} \pm 1.0 \pm 0.5\right) \times 10^{-4}$ and $\mathscr{B}\left(B^{0} \rightarrow D^{-} D_{s 0}^{*+}(2317)\right) \times \mathscr{B}\left(D_{s 0}^{*+}(2317) \rightarrow D_{s}^{+} \pi^{0}\right)=(10.0 \pm 1.2 \pm 1.0 \pm 0.5) \times 10^{-4}$, where the first errors are statistical, the second are systematic and the third are from $D$ and $D_{S}^{*}$ branching fractions. In addition, negative results were reported on a search for hypothetical neutral $\left(Z^{0}\right)$ and doubly charged $\left(Z^{++}\right)$isospin partners of the $D_{s 0}^{*+}(2317)$.

In the second article are reported results of a Dalitz analysis of the three-body $\bar{B}^{0} \rightarrow D^{*+} \omega \pi^{-}$ decay. Measurements of decay fractions for the quasi-two-body $\bar{B} \rightarrow D_{1}(2430)^{0} \omega, \bar{B}^{0} \rightarrow$ $D_{1}(2420)^{0} \omega, \bar{B}^{0} \rightarrow D_{2}(2460)^{0} \omega$ decays as well as $\bar{B}^{0} \rightarrow D^{*+} \rho(1450)^{-}$and combined fraction for $\rho$-like states are presented. An upper limit for the second-class current is also presented. The fractions of longitudinal polarizations are obtained and partial wave fractions of the $D^{* *}$ states are measured. The measurements also show evidence of nontrivial final-state interaction phases for the resonant amplitudes.

In the third article updated results are presented of the $\chi_{c 1}$ and $\chi_{c 2}$ inclusive production in $B$ decays. Exclusive reconstruction was also performed of $B$ decays including a $\chi_{c J}(J=1,2)$ in the final state, as $\chi_{c 1, c 2} K, \chi_{c 1, c 2} K \pi, \chi_{c 1, c 2} \gamma K \pi, \chi_{c 1, c 2} K \pi \pi$ and $\chi_{c 1, c 2} K \pi \pi \pi$.

The European Physical Society Conference on High Energy Physics

22-29 July 2015

Vienna, Austria

${ }^{*}$ Speaker. 


\section{1. "Measurements of $B \rightarrow D D_{s 0}(2317)$ decay rates and a search for isospin partners of the $D_{s 0}^{*+}(2317)$ "}

The $D_{s 0}^{*+}(2317)$ meson was first observed by BaBar as a narrow peak in the $D_{s}^{+} \pi^{0}$ invariant mass spectrum produced in inclusive $e^{+} e^{-} \rightarrow D_{s}^{+} \pi^{0} X$ annihilation processes [1] and confirmed by CLEO [2]. Its production in the $B$ meson decay processes $B \rightarrow \bar{D} D_{s 0}$ (2317) was subsequently established by both Belle [3] and BaBar [4]. Although it is generally considered to be a conventional $I\left(J^{P}\right)=0\left(0^{+}\right)$P-wave $c \bar{s}$ meson, its mass $M_{D_{s 0}^{*+}(2317)}=2317.8 \pm 0.6 \mathrm{MeV}$ [5] is the same as the peak mass of its non-strange counterpart, the $0^{+} \mathrm{P}$-wave $c \bar{q}(q=u$ or $d) D_{0}^{*}$ with mass $M_{D_{0}^{*}}=2318 \pm 29 \mathrm{MeV}$, in spite of the fact that the mass of the $s$-quark is $\sim 100 \mathrm{MeV}$ above that of either of the $q$-quarks. A $c \bar{s}$ meson with mass below the $2358.6 \mathrm{MeV}$ threshold would decay via the isospin-violating process $D_{s 0}^{*+}(2317) \rightarrow D_{s}^{+} \pi^{0}$ or the electromagnetic process $D_{s 0}^{*+}(2317) \rightarrow D_{s}^{+} \gamma$ and, thus, have a narrow natural width. This is consistent with experimental measurements, which have established a 95\% CL upper limit on the total width of $\Gamma_{D_{s 0}^{*+}(2317)} \leq 3.8 \mathrm{MeV}$ [5].

We conclude that an $I=1 ; I_{z}=0$ assignment for the $D_{s 0}^{*+}(2317)$ cannot be ruled out and claim, in fact, that an $I=1$ diquark-diantiquark interpretation is favored by some existing data. If this were the case, doubly charged $I_{z}=1\left(Z^{++}\right)$and neutral $I_{z}=-1\left(Z_{0}\right)$ partners of the $D_{s 0}^{*+}(2317)$ with mass within $\sim \pm 10 \mathrm{MeV}$ of $M_{D_{s 0}^{*+}(2317)}$ should exist. Since the $Z^{++}$and $Z^{0}$ would be charmed mesons with $I=1$ and $S=1$, they would necessarily have a minimal quark content of $c \bar{s} u \bar{d}$ and $c \bar{s} d \bar{u}$, respectively.

\subsection{The $B \rightarrow \bar{D} D_{s 0}^{*+}(2317) ; D_{s 0}^{*+}(2317) \rightarrow D_{s}^{+} \pi^{0}$ results}

The number of $B^{0} \rightarrow D D_{s 0}^{*+}(2317) ; D_{s 0}^{*+}(2317) \rightarrow D_{s}^{+} \pi^{0}$ signal events was determined in the data by applying the three-dimensional fit described above to the selected $\bar{D}=D$ event sample. In this fit, the rms widths of the $M_{b c}, M\left(D_{s}^{+} \pi^{0}\right)$ and $\delta E$ signal functions are kept fixed at their MCdetermined values. Figure 1 shows the results of the fit to the $M_{b c}$ (left), $M\left(D_{s}^{+} \pi^{0}\right)$ (center) and $\delta E$ (right) projections of events that are in the signal regions of the other two quantities. The curves in each plot show the results of the fit, which returns a signal yield of $N_{\text {evt }}=102.6_{-12.0}^{+12.7}$ events. The signal significance is $9.9 \sigma$.

\subsection{Search for $Z^{++} \rightarrow D_{s} \pi^{+}$and $Z^{0} \rightarrow D_{s} \pi$}

For the search for the doubly charged $Z^{++}$and neutral $Z^{0}$, we look for evidence for $Z^{++} \rightarrow$ $D_{s} \pi^{+}$and $Z \rightarrow D_{s} \pi$ signals in the $B \rightarrow D D_{s} \pi$ and $B^{0} \rightarrow \overline{D^{0}} D_{s} \pi^{-}$decay channels, respectively, by applying the selection criteria discussed above with the replacement of the selected $\pi^{0}$ with a $\pi^{+}$(for $Z^{++}$) or $\pi$ (for $Z^{0}$ ). Here, for events with multiple $D$ and/or $D_{s}$ track combinations, those were selected which have a measured invariant mass closest to the corresponding PDG values. For $\mathrm{Z}^{++}$signal MC, the number of remaining events with multiple entries is $11.2 \%$ over the full threedimensional range of the likelihood fit; for $Z^{0}$ less than $0.1 \%$ of the remaining events have multiple entries. Figure 2 shows the fit results for the mass bin centered at $M\left(D_{s}^{+} \pi\right)=2317.8 \mathrm{MeV}$ for the $Z^{++}\left(Z^{0}\right)$ search. 


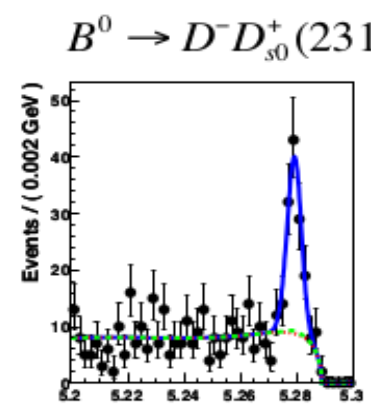

$M_{\mathrm{bc}}(\mathrm{GeV})$

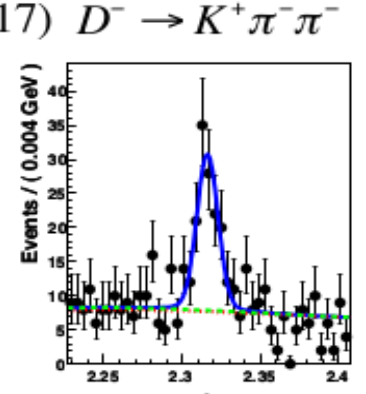

$M\left(D_{s}^{+} \pi^{9}\right)(\mathrm{GeV})$

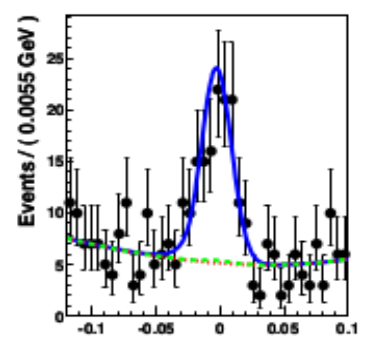

$\Delta E(\mathrm{GeV})$

Figure 1: The $M_{b c}$ (left), $M\left(D_{s} \pi^{0}\right)$ (center) and $\delta E$ (right) distributions for projections of the $B^{0} \rightarrow$ $D^{-} D_{s 0}^{+}(2317)$ candidate events that are in the signal regions of the two quantities not plotted.
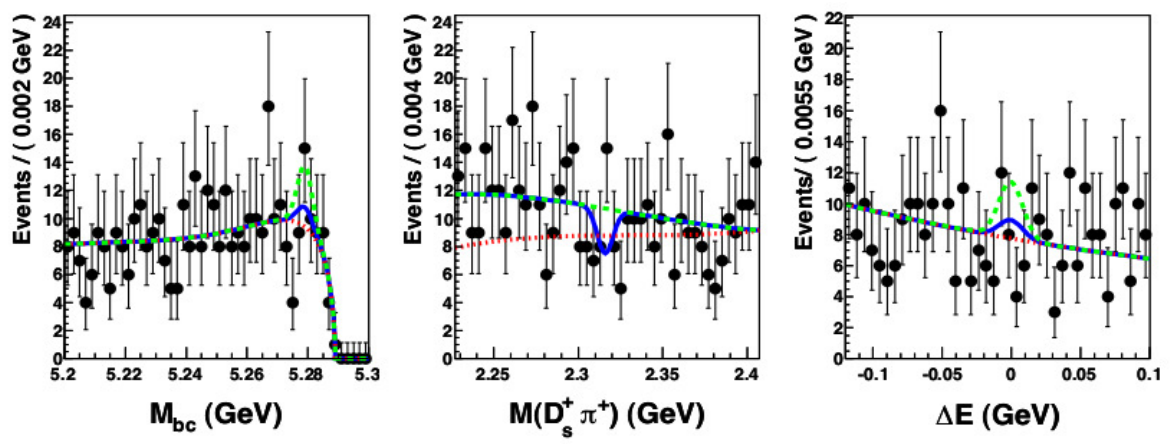

Figure 2: The $M_{b c}$ (left), $M\left(D_{s} \pi\right)$ (center) and $\delta E$ (right) distributions for selected $B^{+} \rightarrow D^{-} D_{s}^{+} \pi^{+}$event candidates for the fit with the signal peak mass restricted to a $5 \mathrm{MeV}$ region.

\subsection{Summary}

We report measurements of the product branching fractions $\mathscr{B}\left(B^{+} \rightarrow \bar{D}^{0} D_{s 0}^{*+}(2317)\right) \times \mathscr{B}\left(D_{s 0}^{*+}(2317) \rightarrow\right.$ $\left.D_{s}^{+} \pi^{0}\right)=\left(7.8_{-1.2}^{+1.3} \pm 1.0 \pm 0.5\right) \times 10^{-4}$ and $\mathscr{B}\left(B^{0} \rightarrow D^{-} D_{s 0}^{*+}(2317)\right) \times \mathscr{B}\left(D_{s 0}^{*+}(2317) \rightarrow D_{s}^{+} \pi^{0}\right)=$ $(10.0 \pm 1.2 \pm 1.0 \pm 0.5) \times 10^{-4}$ These values agree with the existing PDG world=average values [5] and significantly improve upon their precision. In addition, negative results have been reported on a search for hypothetical doubly charged and neutral isospin partners of the $D_{s 0}^{*+}$ and upper limits were provided on the product branching fractions that are more than an order of magnitude smaller than the theoretical predictions [6].

\section{2. "Study of $D^{* *}$ production and light hadronic current in $B^{0} \rightarrow D^{+} \omega \pi^{-}$decay"}

Orbitally excited states of the $D$ meson ( $D$ states) provide a good opportunity to test the Heavy Quark Effective Theory (HQET) [13] and QCD sum rules predictions [8]. The simplest system 
consists of a charm quark and a light antiquark in an orbital angular momentum $L=1$ ( $P$-wave) state. Four such states are expected with spin-parities $J^{P}=0^{+}(j=1 / 2), 1^{+}(j=1 / 2), 1^{+}(j=3 / 2)$ and $2^{+}(j=3 / 2)$, where $j$ is the sum of the light quark spin and angular momentum $L$. All these states have been discovered [9]. They are $D_{0}^{*}(2400), D_{1}(2430), D_{1}(2420)$ and $D_{2}(2460)$.

In this paper an amplitude analysis of the $B^{0} \rightarrow D^{+} \omega \pi^{-}$decay is performed to measure the decay fractions for the $D^{* *}$ states produced via a color-suppressed channel and study their properties. The decay $\bar{B}^{0} \rightarrow D^{*+} \omega \pi^{-}$is expected to proceed predominantly via the $\rho$-like resonances, such as off-shell $\rho(770)^{-}$and $\rho(1450)^{-}$. The structure of the $\rho$-like states is not yet completely clear. Another aim of this study is a test of the factorization hypothesis in the $D^{* *}$ production region.

\subsection{Total branching fraction}

Since the $\bar{B}^{0} \rightarrow D^{*+} \pi^{+} \pi^{-} \pi^{0} \pi^{-}$events observed in Fig. 3 produce a peak in $\Delta E$, the fit is performed separately in the $M\left(\pi^{+} \pi^{-} \pi^{0}\right)$ signal and sideband regions. MC simulation shows that these events have the same shape as the correctly reconstructed component.

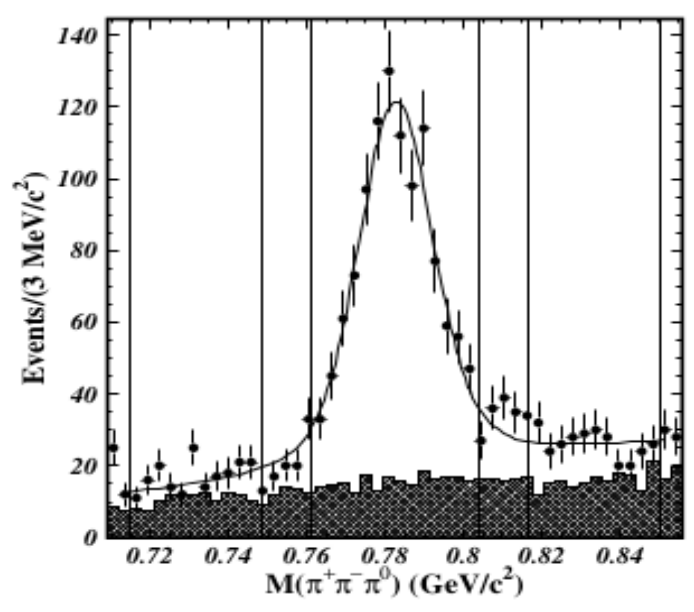

Figure 3: $M\left(\pi^{-} \pi^{+} \pi^{0}\right)$ distribution of the $B^{0} \rightarrow D^{+} \omega \pi^{-}$candidates in the $\Delta E$ signal region (points with error bars) and sideband (hatched histogram).

\subsection{Amplitude analysis}

To study the resonant structure of the $\bar{B}^{0} \rightarrow D^{*+} \omega \pi^{-}$decay, an amplitude analysis was performed. Using an unbinned likelihood method, the data were simultaneously fitted in the sixdimensional phase space according to [12]. Two sets of kinematic variables were defined:

$\left(M^{2}(\omega \pi), \cos \theta_{1}, \phi_{1}, \cos \beta_{1}, \psi_{1}\right.$ and $\left.\cos \xi_{1}\right)$ and $\left(M^{2}(\omega \pi), \cos \theta_{2}, \phi_{2}, \cos \beta_{2}, \psi_{2}\right.$ and $\cos \xi_{2}$, corresponding to the $\omega \pi$ and $D^{* *}$ production, respectively. The masses $M(\omega \pi)$ and $M(D \pi)$ are the invariant masses of the $\omega \pi$ and $D \pi$ combinations. The polar $\theta_{1}\left(\theta_{2}\right)$ angle describes the orientation of the normal to the $\omega$ decay plane relative to the $\omega \pi(D \pi)$ direction in the $\omega$ rest frame and the azimuthal $\phi_{1}\left(\phi_{2}\right)$ angle defines the orientation of the $B$-decay plane relative to the plane including the $\omega \pi\left(D^{*} \pi\right)$ direction and orthogonal to the $\omega$ decay plane in the $\omega$ rest frame. The polar $\beta_{1}\left(\beta_{2}\right)$ angle defines the direction of the $D^{0}$ relative to the $\omega \pi\left(D^{*} \pi\right)$ direction in the $D^{+}$rest frame and 

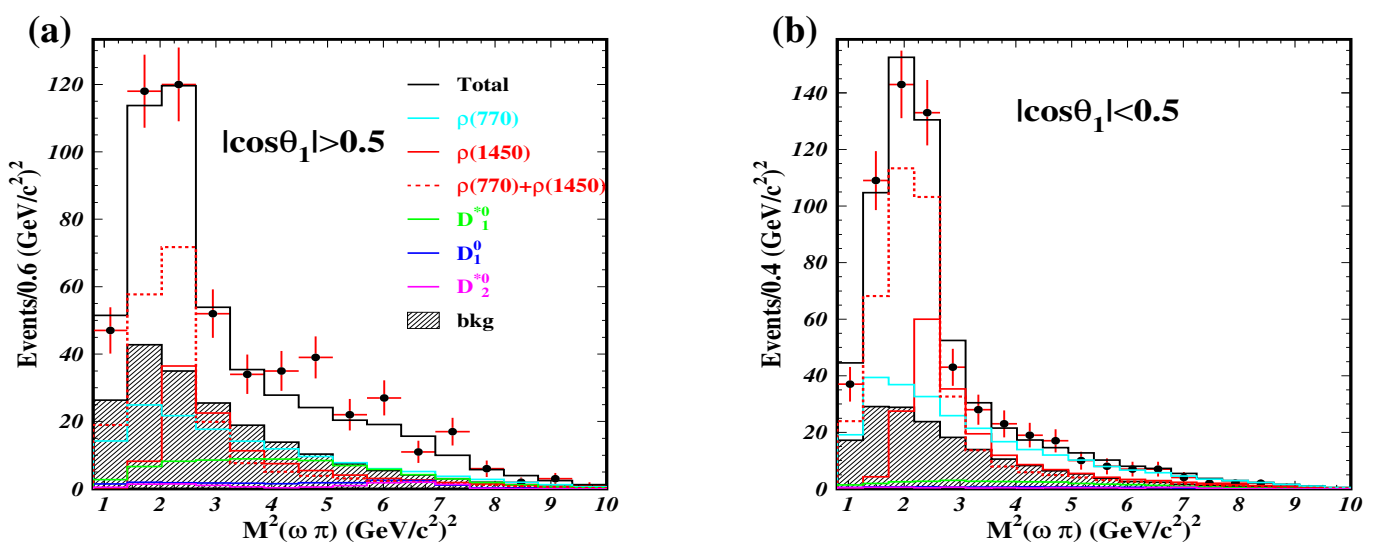

Figure 4: Distribution of $M^{2}(\omega \pi)$ variables for $D^{+} \omega \pi$ candidates in two different subregions of the signal region (points with error bars), defined by $\left|\cos \theta_{1}\right|>0.5\left(D\right.$ enriched) and $\left|\cos \theta_{1}\right|<0.5(D$ depleted).

azimuthal $\psi_{1}\left(\psi_{2}\right)$ angle defines the orientation of the $B$-decay plane relative to the plane including the $D^{0}$ and $\omega \pi\left(D^{*} \pi\right)$ flight directions in the $D$ rest frame. The polar angle $\xi_{1}\left(\xi_{2}\right)$ is the angle between the $D^{+}$and $\omega$ flight directions in the $\omega \pi\left(D^{*} \pi\right)$ rest frame. The quantity $\cos \xi_{1}$ is related to the $M^{2}(D \pi)$, whereas the $\cos \xi_{2}$ is related to $M^{2}(\omega \pi)$.

\subsection{Conclusion}

This analysis is devoted to the study of the three-body $B^{0} \rightarrow D^{+} \omega \pi^{-}$decay. The total branching fraction $B=(2.31 \pm 0.11$ (stat. $) \pm 0.14$ (syst. $)) \times 10^{3}$ has been obtained, consistent within errors with the CLEO [10] and BaBar [11] measurements but with a slightly smaller central value. A full amplitude analysis of the final state has been performed.

\section{3. "Study of inclusive and multi-body $B$ decays into $\chi_{c 1}$ and $\chi_{c 1}$ "}

In two-body $B$ meson decays, the daughter mesons can be directly generated by a quark current carrying the appropriate parity and the flavor quantum numbers. The corresponding contribution to a decay amplitude factorizes into the product of two current matrix elements and this picture is called factorization. On the basis of factorization, $\chi_{c 1}$ (vector $1^{+}$) couples to the $V-A$ operator which results in a proper matrix element. On the other hand, since $\chi_{c 2}$ is a tensor $\left(2^{+}\right)$, it does not couple to vector or axial-vector operator, $\left\langle\chi_{c 2}\left|(\bar{c} c)_{V-A}\right| 0\right\rangle=0$ in the factorization limit. From this it can be said that decay of $B \rightarrow \chi_{c 1} K$ should be favored while $\chi_{c 2} K$ should be disfavored [13]. After taking into account the next-to-leading order (NLO) corrections [14], resscattering effects [15], $B \rightarrow \chi_{c 2} K$ decays are allowed but highly suppressed. A recent study performed at Belle [16] has seen the $B^{ \pm} \rightarrow \chi_{c 2} K^{ \pm}$decay mode with a $3.6 \sigma$ significance and measured the ratio of its yield with respect to $B^{ \pm} \rightarrow \chi_{c 1} K^{ \pm}$as:

$$
\frac{N\left(B^{ \pm} \rightarrow \chi_{c 2} K^{ \pm}\right)}{N\left(B^{ \pm} \rightarrow \chi_{c 1} K^{ \pm}\right)}=\frac{33 \pm 11}{2308 \pm 52}=1.4 \pm 0.4 \%
$$

Extrapolating this in a naive manner, one may expect the same order of suppression in the total yield of $\chi_{c 2}$ in comparison to $\chi_{c 1}$ in inclusive production in $B$ decays. However, a relatively large 
inclusive production of $\chi_{c 2}$ has been seen in the $B$ decays. An early study performed by Belle [17] with $30 \mathrm{fb}^{1}$ found that

$$
\frac{N\left(B^{ \pm} \rightarrow \chi_{c 2} K^{ \pm}\right)}{N\left(B^{ \pm} \rightarrow \chi_{c 1} K^{ \pm}\right)}=\frac{611 \pm 76}{2529 \pm 127}=24.2 \pm 3.2 \%
$$

This means that inclusive production of $\chi_{c 2}$ in $B$ decays is relatively large in spite of the fact that two-body $B$ decays into $\chi_{c 2}$ are highly suppressed.

\subsection{Branching fraction measurement}

To identify the signal, the $J / \psi \gamma$ invariant mass $M_{J / \psi \gamma}$ distribution has been used and the signal yield has been extracted from a binned extended maximum likelihood fit. The signal of $\chi_{c J}$ is described by a double-sided Crystal Ball function [18].

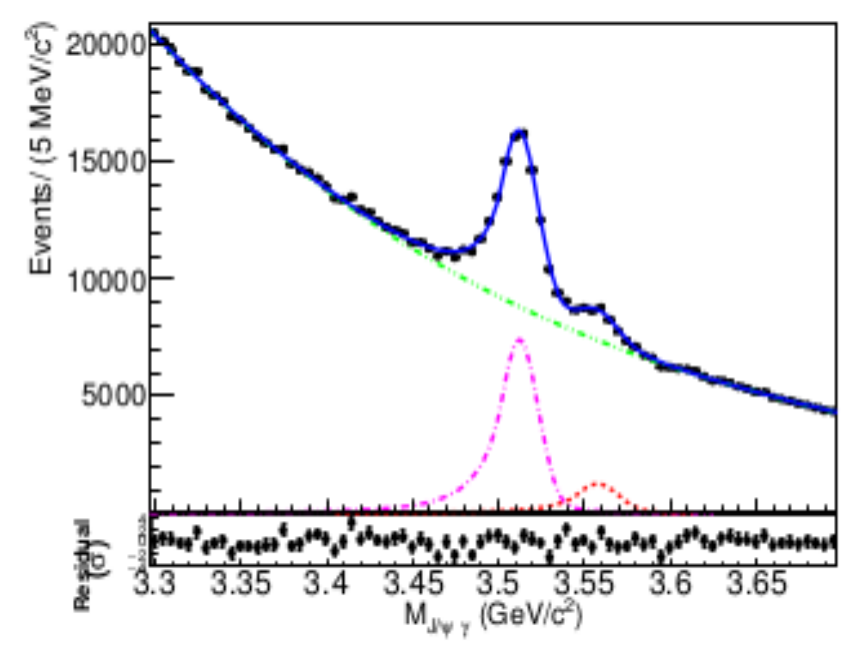

Figure 5: $M_{J / \psi \gamma}$ distribution of the $B \rightarrow \chi_{c J}\left(\rightarrow J / \psi\left(\rightarrow l^{+} l\right) \gamma\right) X$ decays in data. The curves show the signal (magenta dash-dotted for $\chi_{c 1}$ and red dashed for $\chi_{c 2}$ ) and the background component (violet dash doubledotted for combinatorial) as well as the overall fit (blue solid). The lower plot shows the pull of the residuals with respect to the fit.

Figure 5 shows the fit of the $M_{J / \psi \gamma}$ distribution for $B \rightarrow \chi_{c 1} X$ and $B \rightarrow \chi_{c 2} X$ decays in the range of $[3.297,3.697] \mathrm{GeV} / \mathrm{c}^{2}$. A yield of $51353 \pm 614$ was obtained for events for the $\chi_{c 1}$ peak and $9651 \pm 446$ events for the $\chi_{c 2}$ peak, where the errors are statistical only.

\subsection{Summary}

The branching fractions $\mathscr{B}\left(B \rightarrow \chi_{c 1} X\right)$ and $\mathscr{B}\left(B \rightarrow \chi_{c 2} X\right)$ were measured to be $(3.29 \pm 0.04 \pm$ $0.26) \times 10^{3}$ and $(0.97 \pm 0.05 \pm 0.11) \times 10^{3}$, respectively, where the first (second) error is statistical (systematic). After subtracting the $\psi^{\prime}$ feed-down contribution, we get the direct branching fractions $\mathscr{B}\left(B \rightarrow \chi_{c 1} X\right)$ and $\mathscr{B}\left(B \rightarrow \chi_{c 2} X\right)$ to be $(3.03 \pm 0.04 \pm 0.21) \times 10^{3}$ and $(0.70 \pm 0.05 \pm 0.07) \times 10^{3}$, respectively, where the first (second) error is statistical (systematic). Here, a systematic uncertainty 
dominates the measured branching fraction. This is the first observation of the $B^{0} \rightarrow \chi_{c 2} \pi^{-} K^{+}$ decay mode with $206.34 \pm 24.7$ signal events with a $8.7 \sigma$ significance. We report the first observation of $B^{+} \rightarrow \chi_{c 2} \pi^{-} K_{S}^{0}$ decay mode having $76.38 \pm 14.7$ signal events with a significance of $4.6 \sigma$. First observation of $B^{+} \rightarrow \chi_{c 1} \pi^{+} \pi^{-} K^{+}\left[B^{+} \rightarrow \chi_{c 1} \pi^{+} \pi^{-} K^{+}\right]$with a measured branching fraction of $(3.72 \pm 0.17 \pm 0.24) \times 10^{4}\left[(1.33 \pm 0.17 \pm 0.08) \times 10^{4}\right]$. In other four body decay $B^{0} \rightarrow \chi_{c 1} \pi^{+} \pi^{-} K_{S}\left[B^{0} \rightarrow \chi_{c 1} \pi^{0} \pi^{-} K^{+}\right]$was also observed for the first time with a significance of $7.1 \sigma(6.5 \sigma)$.

\section{References}

[1] B. Aubert et al. (BaBar Collaboration), Phys. Rev. Lett. 90, 242001 (2003).

[2] D. Besson et al. (CLEO Collaboration), Phys. Rev. D 68, 032002 (2003).

[3] P. Krokovny et al. (Belle Collaboration), Phys. Rev. Lett. 91, 262002 (2003).

[4] B. Aubert et al. (BaBar Collaboration), Phys. Rev. Lett. 93, 181801 (2004).

[5] J. Beringer et al (Particle Data Group), Phys. Rev. D 86, 010001 (2012).

[6] A. Hayashigaki and K. Terasaki, Prog. Theor. Phys. 14, 1191 (2005).

[7] M. Neubert, Phys. Rept. 245, 259 (1994).

[8] N. Uraltsev, Phys. Lett. B 501, 86 (2001).

[9] K.A. Olive et al. (Particle Data Group), Chin. Phys. C 38, 090001 (2014).

[10] J.P. Alexander et al. (CLEO Collaboration), Phys.Rev. D 64, 092001 (2007).

[11] B. Aubert et al. (BaBar Collaboration), Phys. Rev.D74, 012001 (2006).

[12] We assume equal rates for $\Upsilon(4 S) \rightarrow B^{+} B^{-}$and $\Upsilon(4 S) \rightarrow \bar{B}^{0} B^{0}$, and $N_{B}$ is equal to the number of $B$ pairs produced.

[13] M. Neubert and B. Stech, Adv. Ser. Direct. High Energy Phys. 15, 294-344 (1998) (arXiv:9705292v2 [hep-ph]).

[14] M. Beneke and L. Vernazza, Nuclear Physics B 811, 155 (2009) and references therein.

[15] P. Colangelo, F.De. Fazio and T.N. Pham, Phys. Lett. B 542, 71 (2002) and references therein.

[16] V. Bhardwaj et al., (Belle Collaboration), Phys. Rev. Lett. 107, 091803 (2011); "Study of $B \rightarrow(J / \psi \gamma) K$ and $B \rightarrow(\psi(2 S) \gamma) K^{\prime \prime}$, Internal Belle Note 1123.

[17] K. Abe et al., (Belle Collaboration), Phys. Rev. Lett. 89, 011803 (2002); S. Schrenk et al., "Observation of Inclusive $B \rightarrow \chi_{c 2}$ ", Internal Belle Note 461 .

[18] T. Skwarnicki, Ph.D. thesis, Institute for Nuclear Physics, Krakow, 1986; DESY Internal Report No. DESY F31-86-02, 1986. 